\title{
Screening of differentially expressed protein kinases in bone marrow endothelial cells and the protective effects of the p38a inhibitor SB203580 on bone marrow in liver fibrosis
}

\author{
BO GAO, WANG SUN, XIANZHI MENG, DONGBO XUE and WEIHUI ZHANG \\ Department of General Surgery, The First Affiliated Hospital of Harbin Medical University, \\ Harbin, Heilongjiang 150001, P.R. China
}

Received June 29, 2015; Accepted August 18, 2016

DOI: $10.3892 / \mathrm{mmr} .2016 .5837$

\begin{abstract}
Hematological abnormalities are frequently observed in patients with liver cirrhosis (LC). A previous study demonstrated that the apoptosis and damage of endothelial cells could cause the hematological abnormalities in LC. Protein kinases are one of the most important factors that regulate cell behavior, and are potential therapeutic targets for the treatment of a number of diseases. In a previous study, whole genome profiling was used to identify differentially expressed genes in human bone marrow endothelial cells treated with serum from 26 patients with LC. From this data set, the present study identified 14 differentially expressed kinase genes in human bone marrow endothelial cells in LC from the microarray data, including p38a, AKT1 and PDK1. Pathway analysis revealed that these kinase genes were enriched in certain important LC-associated pathways (e.g. MAPK and WNT signaling pathway). Literature mining revealed that $\mathrm{p} 38 \mathrm{a}$ was associated with bone marrow apoptosis; therefore, p38a and its inhibitor, SB203580, were selected as potential therapeutic targets in the present study. The results of hematoxylin-eosin and Masson's trichrome staining of livers from a rat model of liver fibrosis (LF) that underwent ligation of the bile duct demonstrated that SB203580 reduced the degree of LF. In addition, SB203580-treated rats with LF demonstrated a significantly higher number of platelets when compared with the untreated group. Terminal deoxynucleotidyl transferase dUTP nick end labeling (TUNEL) analysis indicated that apoptosis of bone marrow tissue in rats with LF was inhibited by SB203580. In addition, the results from the
\end{abstract}

Correspondence to: Professor Weihui Zhang or Professor Dongbo Xue, Department of General Surgery, The First Affiliated Hospital of Harbin Medical University, 23 You Zheng Street, Harbin, Heilongjiang 150001, P.R. China

E-mail: zhangweihui626@hotmail.com

E-mail: xue9971@sina.com

Key words: protein kinases, bone marrow, liver fibrosis, p38a, SB203580 immunohistochemical analysis demonstrated that SB203580 reduced the expression of von Willebrand factor and caspase 3 in the bone marrow of rats with LF. In conclusion, the results from the present study indicate that the p38a kinase inhibitor, SB203580, may exhibit a protective effect on bone marrow tissues in rats with LF. This suggests that protein kinases and their inhibitors may present novel therapeutic strategies for the treatment of hematological abnormalities in patients with LC.

\section{Introduction}

A reduction in the number of blood cells, including red blood cells, leukocyte and platelets, is a common complication of liver cirrhosis (LC), and may exacerbate the progression of the disease and severely affect the patient's quality of life (1). The mechanisms underlying the observed reduction in the number of blood cells in LC are unknown, however, several mechanisms have been proposed for this anomaly. For instance, hypersplenism and reduced liver thrombopoietin leads to platelet deficiency (2). In addition, portal hypertension may lead to gastrointestinal bleeding, hemolysis and reduced hematopoietic substances, such as iron and folic acid, which may induce anemia (3). Furthermore, hepatitis viruses, excessive alcohol consumption and the intake of certain drugs, including methotrexate and amiodarone, often lead to LC (4,5). If bone marrow production is suppressed, these factors may lead to deficiencies in bone marrow function, which may subsequently affect the function of the hematopoietic system $(6,7)$. A previous study demonstrated that bone marrow endothelial cells (BMECs) derived from rats with liver fibrosis (LF) exhibited ultrastructural changes in vivo, and in vitro results demonstrated that the serum of patients with LC may induce apoptosis in BMECs $(8,9)$.

BMECs are an important component of the hematopoietic microenvironment, where they generate hematopoietic stem cells and serve an important role in regulating the self-renewal, differentiation, homing and migration of hematopoietic stem cells (10-12). Therefore, damage to the bone marrow microenvironment during LC by serum inhibitory factors such as endotoxin and inflammatory cytokines, may subsequently result in damage to hematopoietic stem cells. Thus, determining the mechanisms underlying the destructive actions of 
LC on the bone marrow microenvironment, and the identification of an effective drug therapy that inhibits this process, is important for the study of hematological abnormalities during LC, as well as for the clinical treatment of LC.

Previous studies have demonstrated that protein kinases, which are intermediate molecules in signal transduction pathways, can regulate the activity of metabolic enzymes or the expression of genes by phosphorylating target proteins (13). Protein kinases are one of the most important regulatory factors of cell behavior, and are associated with almost all cellular functions. The central role of protein kinases in controlling cellular behavior demonstrates their potential as a therapeutic target for a number of diseases, including cancer, inflammation and eye diseases (14). Thus, protein kinases have been widely studied as potential therapeutic targets. In addition, with the increase in the number of studies investigating protein kinases as therapeutic targets for different diseases, the development of novel kinase inhibitors is increasing rapidly. To date, 30 kinase inhibitors have been approved by the US Food and Drug Administration for clinical treatment or testing, and these developments have promoted the advancement of laboratory results to clinical practice (15).

In the present study, whole genome microarray results obtained from previous studies were used to screen for differentially expressed kinase genes in BMECs treated with serum derived from patients with LC and normal healthy controls $(8,9)$. Bioinformatics tools were used to predict the functions of differentially expressed kinases, and the signaling pathways that they may regulate. Finally, a kinase inhibitor was used to inhibit the activity of a candidate protein kinase in a rat model of LF, in order to determine its effect on bone marrow tissue function.

\section{Materials and methods}

Bioinformatics analysis. In a previous study (9), the sera from 26 patients with LC and 10 healthy volunteers were used to treat BMECs for $48 \mathrm{~h}$, resulting in identification of 1,872 differentially expressed genes by screening whole genome microarray chips, with 1,106 overexpressed genes and 766 underexpressed genes. Reverse transcription-quantitative polymerase chain reaction analysis was used to verify the results of the whole genome microarray chips in a previous study (9). Patient clinical data, such as the number of blood cells in the 26 patients with LC, was described previously (9). In the present study, pathway and gene ontology analyses of these differentially expressed genes were performed using the Kyoto Encyclopedia of Genes and Genomes (KEGG; http://www.genome.jp/kegg/pathway.html) and the Database for Annotation, Visualization and Integrated Discovery (DAVID; https://david.ncifcrf.gov/) databases in order to identify their predicted functions and the signaling pathways that they may regulate. Differentially expressed protein kinase genes were identified using the Kinase Substrate Database (http://kinasource.co.uk/Database/substrates.html), and were selected for further screening. The search terms used to generate the protein kinase list was obtained from kinase library (https://www.cellsignal.com/common/content/content. jsp?id=kinases). The predicted pathways of differentially expressed kinase genes were identified using KEGG, and
Cytoscape software (version 2.8.2; http://www.cytoscape.org/) was used to generate a kinase-pathway network. ActivePerl software (version 5.16.2; http://www.activestate.com/active$\mathrm{perl} /$ ) was used to retrieve literature data from the National Center for Biotechnology Information (U.S. National Library of Medicine, Bethesda, MD, USA) PubMed database (http://www.ncbi.nlm.nih.gov/pubmed). The search results included the titles and abstracts of publications, and the search keywords were the names of the differentially expressed genes and 'bone marrow apoptosis' (for example, 'AKT and bone marrow apoptosis').

Animal model. All procedures involving animals, including animal feeding, operation and sacrifice, were performed after receiving the approval of the institutional ethics review board of the First Affiliated Hospital of Harbin Medical University (Harbin, China). A total of 36 healthy male Wistar rats (weight, 230-250 g; age, 7 weeks; purchased from Changchun Yisi Laboratory Animal Co., Ltd., Changchun. China) were used in the present study, maintained on a 12-h light/dark cycle in a temperature- and humidity-controlled room with access to water and food ad libitum. The rats were divided into a sham group (control), consisting of 13 rats with a bile duct that was separated but not ligated, and an LF group, consisting of 23 rats with a bile duct that was ligated. To achieve this, the rats were weighed, and $10 \%$ chloral hydrate was injected intraperitoneally $(0.3 \mathrm{ml} / 100 \mathrm{~g}$ body weight) to anesthetize the rat, which normally required $2-3 \mathrm{~min}$. The animal was then fixed on the experiment bench, and the hair from the abdomen was shaved to prepare the skin, which was disinfected and surgical drapes were placed over the abdomen. A 2-3 cm incision was made along the midline section of the abdomen below the xiphoid, and retractors were used to open the abdominal walls and fix them in place. The abdominal organs were examined and were observed to be normal. The duodenum at the pylorus was then identified, and in the middle section of the duodenum, the light yellow bile duct $4 \mathrm{~cm}$ in length and with a diameter of $2 \mathrm{~mm}$ was observed. A glass dissecting needle was used to isolate $1 \mathrm{~cm}$ of bile duct from its upper section. For the LF group, a 3-0 thread was used to ligate the short duct at the proximal and distal ends, and the duct was cut in the middle. For the control group, there was no further treatment after the bile duct was isolated. The abdomen was closed, and a 2-0 thread was used as a simple continuous suture of the peritoneum and muscle, which was applied at an interval of $0.5 \mathrm{~cm}$, and as a discontinuous suture of the skin. Following surgery, $5 \mathrm{ml}$ of saline solution was administered in the tail vein for rehydration. The rats were placed in a warm location until they recovered, which occurred after $\sim 3 \mathrm{~h}$. The rats were provided with food and water ad libitum, and their condition was monitored every day including mental condition, feeding state, and skin and urine color. At 3 weeks following surgery, three rats were selected at random from each group for liver pathological examinations to verify the status of the rat model.

Aside from the 10 rats in the control group, the 14 rats in the LF group that survived after the model was established, were randomly divided into two subgroups: The kinase inhibitor group, consisting of seven rats that were treated with a kinase inhibitor, and the LF group, consisting of seven rats that were treated with the solvent solution without the kinase inhibitor. 
Based on the bioinformatics analysis results, p38a was selected as the target kinase, and the p38a kinase inhibitor SB203580 (Selleck Chemicals, Houston, TX, USA) was used to perform subsequent experiments. The chemical formula of SB203580 is shown in Fig. 1. The kinase inhibitor group was treated with SB203580 (hereafter referred to as the SB203580 group). To achieve this, $50 \mathrm{mg}$ SB203580 was dissolved in $1 \mathrm{ml}$ dimethyl sulfoxide (DMSO) prior to the addition of $19 \mathrm{ml}$ sterile saline solution to produce a 5\% DMSO inhibitor solution. At 3 weeks following surgery, the LF rats were treated with SB203580 for 7 days. A total of $1 \mathrm{mg}$ inhibitor solution $/ \mathrm{kg}$ body weight was injected intravenously each day into rats in the SB203580 group. The LF and control groups were given an equal volume of 5\% DMSO solution. After 1 week, $10 \%$ chloral hydrate was injected intraperitoneally $(0.3 \mathrm{ml} / 100 \mathrm{~g}$ body weight) to terminally anesthetize the rat. Following anesthesia, the femur and tibia were isolated, and the muscle and tendon were removed, then the rats were sacrificed by bloodletting from the abdominal aorta. Samples were collected for blood analysis, pathological examination of the liver, a bone marrow smear, and apoptosis-associated tests.

Blood counts. Whole blood ( $3 \mathrm{ml})$ from all rats was obtained from the abdominal aorta immediately after the femurs were removed. Whole blood samples were analyzed using a Cell-Dyn 3500 (Abbott Diagnostics, Abbott Park, IL, USA) programmed for rat peripheral blood cells. The absolute number of leukocytes and platelets, and concentration of hemoglobin were determined.

Hematoxylin and eosin $(H \& E)$ staining of the liver. The rat liver was fixed in $10 \%$ neutral formalin for $24 \mathrm{~h}$, followed by dehydration and embedding in paraffin. Sections were prepared using a microtome (Labsun China Co. Ltd., Shanghai, China) to slice the paraffin wax. Slice thickness was set to $7 \mu \mathrm{m}$. The slices were washed in phosphate buffered saline (PBS) for $5 \mathrm{~min}$, and hematoxylin was used to stain the nuclei for $5 \mathrm{~min}$, followed by destaining with hydrochloric acid and ethanol for $30 \mathrm{sec}$. The slices were then washed using tap water and distilled water for $1 \mathrm{~min}$. Eosin staining was performed for $30 \mathrm{sec}$, followed by a $70 \%$ ethanol rinse for $30 \mathrm{sec}$, a $90 \%$ ethanol rinse for $30 \mathrm{sec}$, a $95 \%$ ethanol rinse for $30 \mathrm{sec}$, two $100 \%$ ethanol rinses for 2 min each time and xylene clearing twice for 5 min each time. The stained slices were observed under an inverted light microscope.

Masson's trichrome staining of the liver. Paraffin-embedded slices were dewaxed and rehydrated, before the slices were washed in tap water and distilled water. Regaud's hematoxylin staining solution or Weigert's hematoxylin staining solution was used to stain the nuclei for 5-10 min before washing. The slices were thoroughly washed thoroughly with water. If the tissues were over-stained, they were destained with $1 \%$ hydrochloric acid in ethanol and washed with distilled water. Masson Ponceau-acidic fuchsin staining solution was used to stain the slices for 5-10 min, and 2\% acetic acid was then used to briefly wash the slice. The slices were subsequently destained with a $1 \%$ phosphomolybdic acid aqueous solution for 3-5 min before they were stained with aniline blue or green staining solution for $5 \mathrm{~min}$. The slices were briefly rinsed with

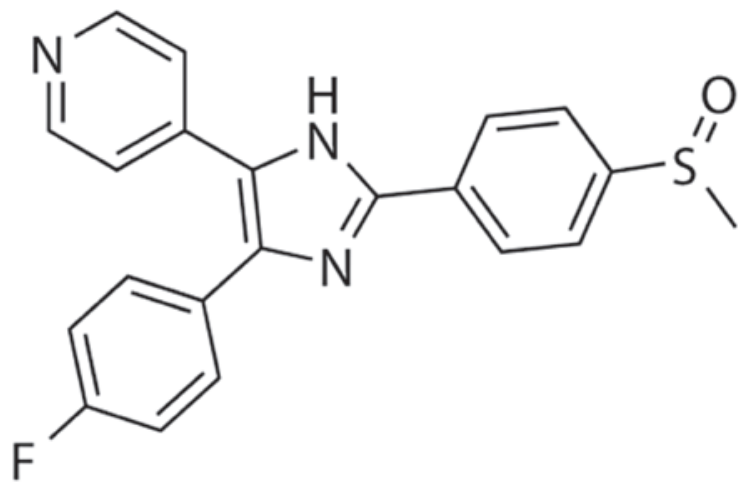

Figure 1. The chemical formula of the p38a inhibitor, SB203580.

a $0.2 \%$ glacial acidic acid solution and then rinsed in $95 \%$ ethanol and absolute ethanol, cleared in xylene and sealed with neutral resin.

Preparation of bone marrow smears. After the rats were anesthetized, the femur and tibia were isolated, and the muscle and tendon were removed. The metaphysis of the long bone was removed, and rat serum was used to repeatedly wash the marrow cavity until no marrow remained. A pipettor was used to mix the bone marrow fluid until uniform. One drop of bone marrow fluid was then placed at one end of a slide, and the frosted end of another glass slide was used to flatten the drop and spread it along the slide. The slide was air-dried at room temperature for subsequent bone marrow staining.

Apoptosis detection in the bone marrow. DNA damage and cell death in rat bone marrow samples was detected using the ApopTag Plus Peroxidase in situ Apoptosis detection kit (cat. no. S7101; EMD Millipore, Billerica, MA, USA) according to manufacturer's instructions. Briefly, tissue sections were deparaffinized and pretreated with Proteinase-K solution (20 $\mu \mathrm{g} / \mathrm{ml})$ at room temperature for $15 \mathrm{~min}$. Endogenous peroxidase activity was quenched using $3 \%$ hydrogen peroxide in PBS at room temperature for $15 \mathrm{~min}$. Following incubation with terminal deoxynucleotidyl transferase at $37^{\circ} \mathrm{C}$ for $1 \mathrm{~h}$, the apoptotic cells were visualized under a bright-field microscope using a diaminobenzidine (DAB)-based detection system supplied with the kit, and sections were counterstained with the methyl green nuclear stain (Trevigen, Gaithersburg, MD, USA). Terminal deoxynucleotidyl transferase dUTP nick end labeling (TUNEL)-positive cells were counted in five high-power fields of view (magnification, $\mathrm{x} 40$ ) selected at random, and counts from three sections for each rat were used for statistical analysis.

Detection of caspase 3 and von Willebrand factor $(v W F)$ in the bone marrow. Immunohistochemical staining was conducted using the streptavidin-peroxidase method (16). Tissue sections were first prepared using the procedures described above. Endogenous peroxidase activity was inhibited by incubating tissue sections in 3\% hydrogen peroxide. Antigen retrieval was achieved by autoclaving tissue samples at $120^{\circ} \mathrm{C}$ for $15 \mathrm{~min}$ in $0.01 \mathrm{M}$ citrate buffer ( $\mathrm{pH}$ 6.0). The sections were incubated overnight at $4^{\circ} \mathrm{C}$ with rabbit polyclonal anti-caspase 3 (dilution, 

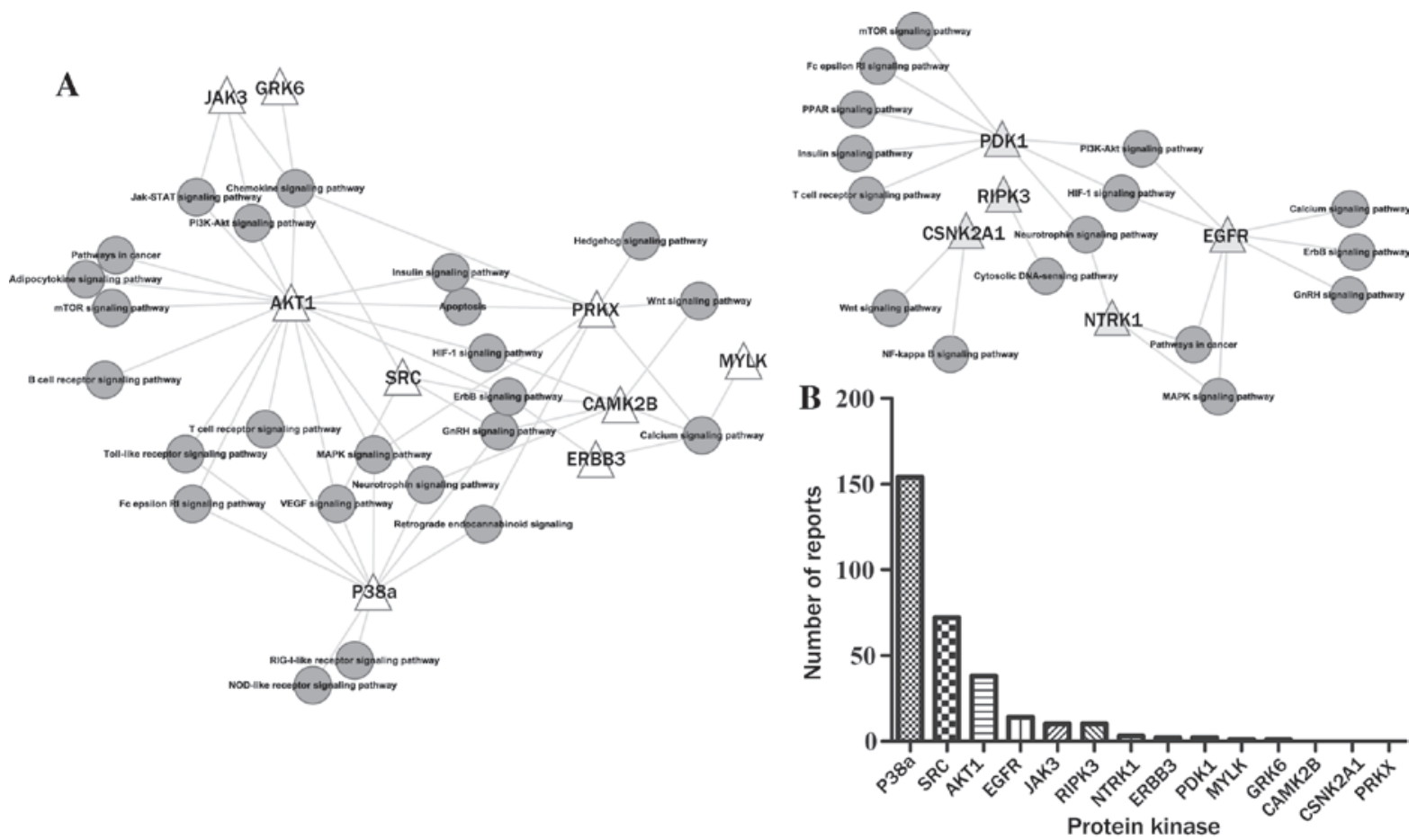

Figure 2. Differentially expressed protein kinases in BMECs treated with serum from patients with LF. (A) Kyoto Encyclopedia of Genes and Genomes (http://www.genome.jp/kegg/pathway.html) pathway analysis of 14 differentially expressed protein kinases. A total of nine protein kinases were overexpressed (JAK3, GRK6, AKT1, PRKX, MYLK, CAMK2B, ERBB3, SRC, p38a) and five were underexpressed (PDPK1, RIPK3, CSNK2A1, EGFR, NTRK1). (B) The number of reports identified regarding individual protein kinases and bone marrow apoptosis (search term, e.g., 'AKT1 + bone marrow apoptosis') as determined using the national center for biotechnology information PubMed (http://www.ncbi.nlm.nih.gov/pubmed/) database. BMECs, bone marrow endothelial cells; LF, liver fibrosis. See the main text for the abbreviations of the kinases.

1:65; cat. no. WL0146; Wanleibio Co., Ltd., Shenyang, China) and anti-vWF (dilution, 1:65; cat. no. sc-365712; Santa Cruz Biotechnology, Inc., Dallas, TX, USA) primary antibodies. The biotinylated goat anti-rabbit IgG secondary antibody (dilution, 1:1,000; cat. no. A0277; Beyotime Co. Ltd., Shanghai, China) was then applied for $30 \mathrm{~min}$ at room temperature. Sections were treated with horseradish peroxidase-labeled streptavidin (Beyotime Co. Ltd.) for $30 \mathrm{~min}$ at $37^{\circ} \mathrm{C}$. Then sections were visualized by 3,30-DAB-tetrahydrochloride horseradish peroxidase color development kit staining (cat. no. P0203; Beyotime Co.Ltd.). The expression of caspase-3 and vWF were quantified using Image-Pro Plus software (Media Cybernetics, Inc., Rockville, MD, USA).

Statistical analysis. Data are expressed as the mean \pm standard deviation. Comparisons between two groups were performed using the Student's $t$-test. Based on information provided by the company that conducted the mRNA microarray analysis (KangChen Bio-tech Inc., Shanghai, China), a $\geq 2$-fold alteration in gene expression was considered to be significant (9). Statistical analyses were performed using SPSS statistics software (version, 15.0; SPSS, Inc., Chicago, IL, USA). P<0.05 was considered to indicate a statistically significant difference.

\section{Results}

Screening of differentially expressed protein kinases in bone marrow endothelial cells during LC. Additional pathway and functional analyses were performed on the 1,106 overexpressed genes and 776 underexpressed genes identified in a previous study, whereby BMECs were treated with serum from patients with LF or normal healthy individuals (9). In the present study, a total of 14 differentially expressed kinase genes were identified through screening, of which nine were overexpressed [Janus kinase 3 (JAK3), G-protein-coupled receptor kinase 6 (GRK6), AKT serine/threonine kinase 1 (AKT1), protein kinase, $\mathrm{X}$-linked (PRKX), myosin light chain kinase (MYLK), calcium/calmodulin-dependent protein kinase type II $\beta$-chain (CAMK2B), Erb-B2 receptor tyrosine kinase 3 (ERBB3), Src and p38a] and five were underexpressed [3-phosphoinositide-dependent kinase 1 (PDPK1), receptor-interacting serine/threonine-protein kinase 3 (RIPK3), casein kinase $2 \alpha-1$ (CSNK2A1), epidermal growth factor receptor (EGFR) and neurotrophic receptor tyrosine kinase 1 (NTRK1)]. In the present study, KEGG pathway analysis demonstrated that these differentially expressed kinases may regulate a number of important signaling pathways (Fig. 2A). The results of the literature mining demonstrated that $>153$ reports regarding p38a and 'bone marrow apoptosis' were identified, which was the greatest number of reports identified when compared with the other kinases that were searched (Fig. 2B). Therefore, p38a was selected as the candidate kinase for further investigation, and the p38a inhibitor SB203580 was employed in subsequent experiments.

Liver fibrosis model. As shown in Fig. 3A, the rat livers in the control group were reddish in color, with a smooth surface and a soft texture. In contrast, rat livers in the LF group exhibited a dull yellow color, and the surface possessed uniform granules with a hard texture when handled. Compared with the 
Table I. Analysis of blood cells in control, LF and SB203580 rats at 4 weeks following surgery.

\begin{tabular}{lcccc}
\hline Group & $\mathrm{n}$ & Hemoglobin $(\mathrm{g} / \mathrm{l})$ & Leukocytes $\left(\mathrm{x} 10^{9} / \mathrm{l}\right)$ & Platelets $\left(\mathrm{x} 10^{9} / \mathrm{l}\right)$ \\
\hline SB203580 & 7 & $153.80 \pm 3.75$ & $16.53 \pm 2.59$ & $487.72 \pm 48.04^{\mathrm{a}}$ \\
LF & 7 & $143.40 \pm 8.26$ & $15.29 \pm 2.65$ & $422.01 \pm 43.80$ \\
Control & 10 & $157.66 \pm 8.19$ & $17.08 \pm 1.47$ & $527.44 \pm 49.77^{\mathrm{a}}$ \\
\hline
\end{tabular}

${ }^{\text {aP }}<0.05$ vs. the LF group. LF, liver fibrosis; control group, sham-operated rats; LF group, rats that underwent ligation of the bile duct; SB203580 group, LF rats that underwent treatment with the p38a inhibitor, SB203580.

control group, livers from the LF group were visibly reduced in volume and weight. In addition, the livers from the LF group were observed to frequently adhere to other organs, and there were difficulties when isolating the organ (Fig. 3A).

As demonstrated by $\mathrm{H} \& \mathrm{E}$ staining, the liver tissue in control rats at three weeks following surgery exhibited a normal structure, and the hepatocytes were intact and aligned in order (Fig. 3B). The liver tissue did not present with lesions, the liver lobular structure was normal, and the hepatic cord exhibited a regular structure without abnormal fibrosis. At 3 weeks following surgery in the LF group, the liver tissue exhibited a disorganized structure, and several striped regions of fibrosis were observed (Fig. 3B). As determined using Masson's trichrome staining, no collagen was observed in the livers from the control group at three weeks following surgery (Fig. 3B). However, in the LF group, collagen fiber hyperplasia was observed, the portal area was expanded, and the over-proliferated fibers extended out from the central vein or portal area (Fig. 3B).

As demonstrated by H\&E staining at 4 weeks following surgery, the livers from LF group rats exhibited an abnormal lobular structure. The presence of fibrous connective tissue hyperplasia, pseudolobule formation and damaged lobular structures were separated and formed hepatocyte groups of different sizes (Fig. 3C). The degree of liver fibrosis in the SB203580 group was markedly reduced compared with that of the LF group. Masson's trichrome staining of liver samples at 4 weeks following surgery demonstrated that collagen fiber hyperplasia was more pronounced in the LF group, with several samples demonstrating fibrous septa formation, and separation of the liver lobules to form pseudolobules. In contrast, the fiber hyperplasia in the SB203580 group was reduced compared with that of the LF group (Fig. 3C).

Analysis of blood cell number. As shown in Table I, analysis of blood cells at 4 weeks following surgery demonstrated that the amount of hemoglobin was $157.66 \pm 8.19 \mathrm{~g} / \mathrm{l}$, the number of white blood cells was $17.08 \pm 1.47 \times 10^{9}$ cells $/ 1$, and the number of platelets was $527.44 \pm 49.77 \times 10^{9}$ cells $/ 1$ in the control group. In the LF group, the amount of hemoglobin was $143.40 \pm 8.26 \mathrm{~g} / \mathrm{l}$, the number of white blood cells was $15.29 \pm 2.65 \times 10^{9}$ cells $/ 1$, and the number of platelets was $422.01 \pm 43.80 \times 10^{9}$ cells/1. In the SB203580 group, the amount of hemoglobin was $153.80 \pm 3.75 \mathrm{~g} / \mathrm{l}$, the number of white blood cells was $16.53 \pm 2.59 \times 10^{9}$ cells $/ 1$, and the number of platelets was $487.72 \pm 48.04 \times 10^{9}$ cells $/ 1$. A comparison of the three groups including comparison between any two groups
A
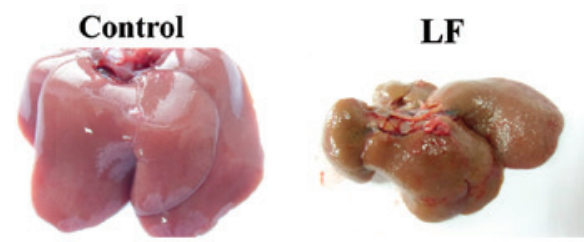

B
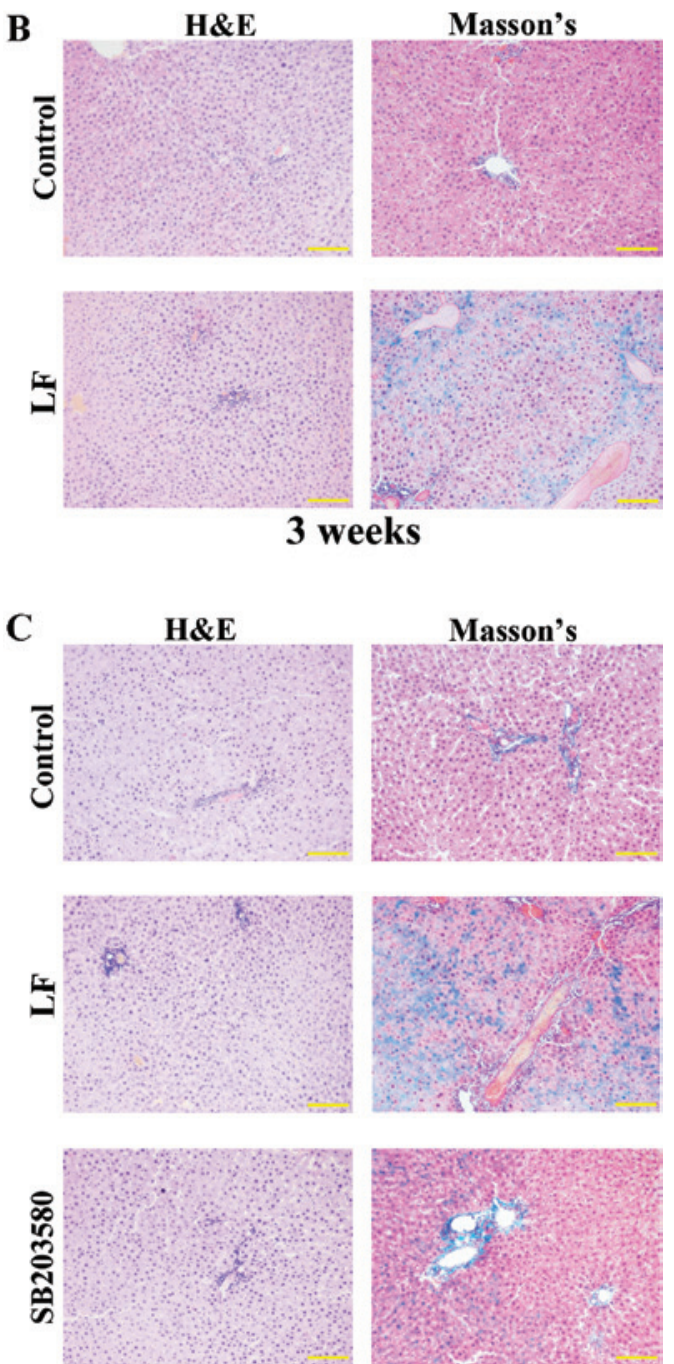

4 weeks

Figure 3. Establishment of a rat LF model and the effect of SB203580 on liver pathology in rats with LF. (A) Photographs of livers obtained from control and LF groups. H\&E staining and Masson's trichrome staining of (B) control and LF rat group livers at 3 weeks following surgery and (C) control, LF and SB203580 rat group livers at 4 weeks following surgery (magnification, x200; scale bars represent $100 \mu \mathrm{m}$ ). LF, liver fibrosis; H\&E, hematoxylin and eosin; control group, sham-operated rats; LF group, rats that underwent ligation of the bile duct; SB203580 group, LF rats that underwent treatment with p38a inhibitor SB203580. 

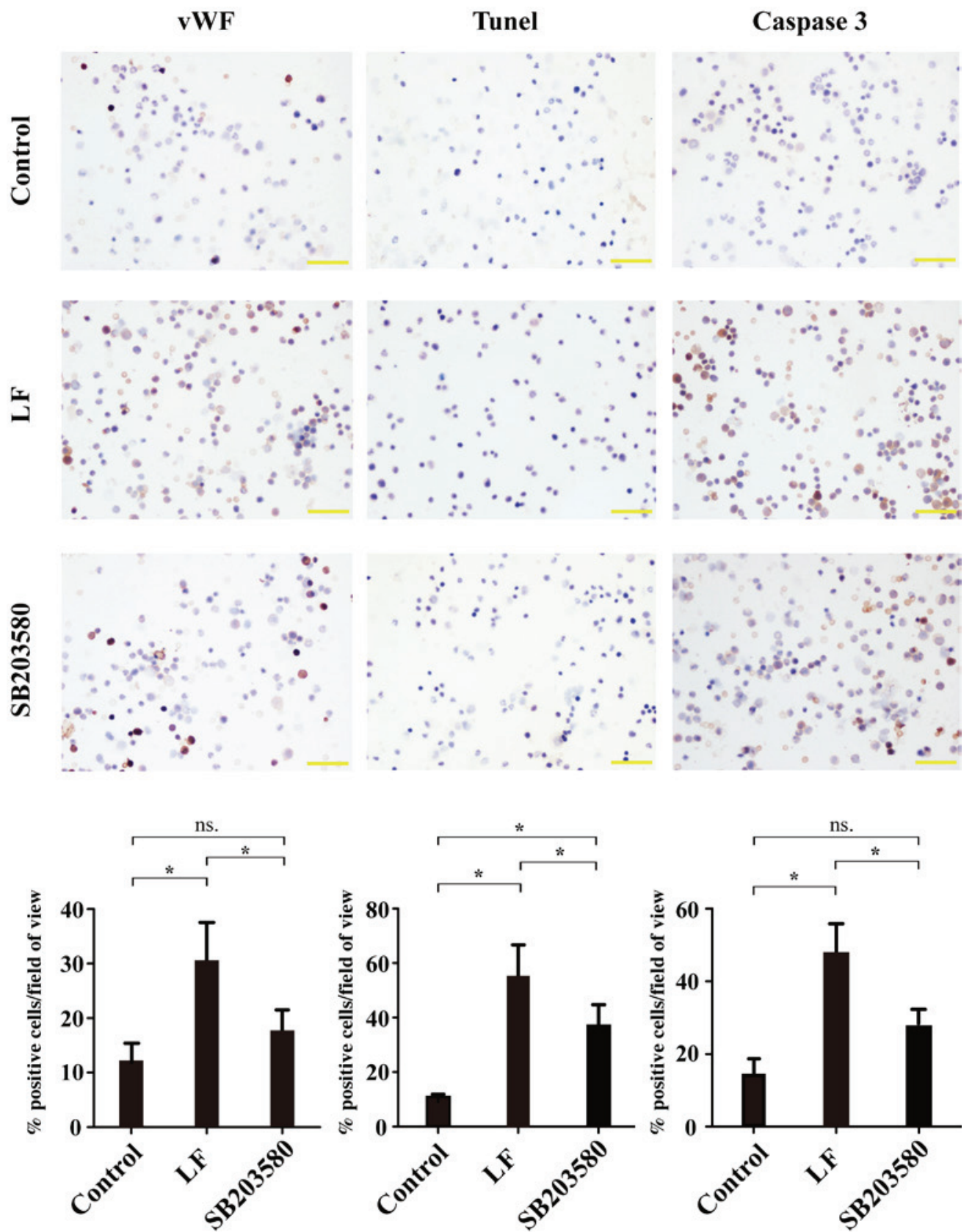

Figure 4. Immunohistochemical analysis of bone marrow smears from control, LF and SB203580 groups for vWF, TUNEL and caspase 3-positive cells. For vWF, the positive expression rate was $12.67 \pm 2.40 \%$ in the control group, $30.86 \pm 6.85 \%$ in the LF group and $17.73 \pm 4.37 \%$ in the SB203580 group. For TUNEL-positive cells, the positive expression rate was $12.57 \pm 2.86 \%$ in the control group, $57.28 \pm 11.03 \%$ in the LF group and $33.61 \pm 9.24 \%$ in the SB203580 group. For caspase 3, the positive expression rate was $13.37 \pm 4.40 \%$ in the control group, $48.13 \pm 13.67 \%$ in LF group and $23.78 \pm 4.80 \%$ in the SB203580 group. Magnification, $\mathrm{x} 400$; Scale bars represent $50 \mu \mathrm{m}$. " $\mathrm{P}<0.05$ vs. control, LF or SB203580 groups as indicated; ns, $\mathrm{P} \geq 0.05$. LF, liver fibrosis; vWF, von Willebrand factor; TUNEL, terminal deoxynucleotidyl transferase dUTP nick end labeling; ns, not significant; control group, sham-operated rats; LF group, rats that underwent ligation of the bile duct; SB203580 group, LF rats that underwent treatment with p38a inhibitor SB203580.

demonstrated that there was no significant difference in the amount of hemoglobin or the number of white blood cells among the groups. However, a significantly higher number of platelets were observed in the SB203580 group and the control group when compared with the LF group (SB203580 group vs. LF group, $\mathrm{P}=0.0303$; control group vs. LF group, $\mathrm{P}<0.0001$ ). No significant difference in platelet number was observed between the SB203580 and the control groups.

vWF expression in bone marrow smear. Positive immunohistochemical staining of $\mathrm{vWF}$ was indicated by the presence of brown-colored cells. The positive expression rate was $12.67 \pm 2.40 \%$ in the control group, $30.86 \pm 6.85 \%$ in the LF group and $17.73 \pm 4.37 \%$ in the SB203580 group (Fig. 4). vWF expression was significantly increased $(\mathrm{P}=0.0122)$ in the $\mathrm{LF}$ group compared with the control group. Compared with the SB203580 group, vWF expression in the LF group was significantly decreased $(\mathrm{P}=0.0488)$, however, no significant difference was observed between the control and SB203580 groups (Fig. 4).

Detection of bone marrow apoptosis. Positive TUNEL detection is indicated by the presence of brown-yellow colored 
cells. As shown in Fig. 4, the positive expression rate was $12.57 \pm 2.86 \%$ in the control group, $57.28 \pm 11.03 \%$ in the LF group and $33.61 \pm 9.24 \%$ in the SB203580 group. Bone marrow apoptosis was significantly higher in the LF group compared with the control group $(\mathrm{P}=0.0024)$. In addition, bone marrow apoptosis was significantly decreased in the SB203580 group compared with the LF group $(\mathrm{P}=0.0197)$, whereas a significant increase in apoptosis was observed between the SB203580 group and the control group ( $\mathrm{P}=0.0464$; Fig. 4).

Caspase 3 expression in the bone marrow. Positive immunohistochemical staining of caspase 3 is indicated by the presence of brown-colored cells. The positive expression rate of caspase 3 was $13.37 \pm 4.40 \%$ in the control group, $48.13 \pm 13.67 \%$ in the LF group and $23.78 \pm 4.80 \%$ in the SB203580 group (Fig. 4). Compared with the control group, caspase 3 expression was significantly increased in the LF group $(\mathrm{P}=0.0138)$. Caspase 3 expression was significantly reduced in the SB203580 group compared with the LF group $(\mathrm{P}=0.0436)$, whereas no significant difference was observed between the SB203580 and the control group (Fig. 4).

\section{Discussion}

In the present study, bioinformatic analysis was performed on the whole genome array data obtained from a previous study (9), and 14 differentially expressed genes that encode protein kinases were observed in BMECs following exposure to serum from patients with LC. Out of these, nine genes were overexpressed, and five genes were underexpressed. Based on these results, combined with the literature-mining results, the p38a protein kinase and its inhibitor, SB203580, were selected for in vivo experiments. The results of the in vivo experiments indicated that the bone marrow apoptotic rate, and the level of caspase 3 and vWF expression in rats with LF, was significantly increased compared with controls. This demonstrated that endothelial cell damage may be increased in rats with LF compared with normal rats. However, following administration of the p38a inhibitor, SB203580, the rate of apoptosis, caspase 3 and vWF expression levels were significantly decreased.

Numerous studies have demonstrated that protein kinases are the most important factors for regulating cell behavior and function. The central role of protein kinases in regulating cell behavior indicates their potential for use as therapeutic targets in cancer, inflammation and numerous additional diseases, which has been studied widely (17-19). Among the methods used to screen target kinases, whole genome arrays provide a significant amount of information about protein kinases, and have become an important method for identifying and analyzing kinases and kinase families. For example, Hofberger et al (20) used whole genome and bioinformatics analysis tools to study the expression and function of the L-type lectin receptor kinase gene family in the Brassicaceae flowering plant family. Draghetti et al (21) used whole genome analysis and determined that polo-like kinase 2 functions to regulate the differentiation of neurons. In the present study, whole genome and bioinformatics analyses were performed to screen for differentially expressed genes that encode protein kinases in BMECs following exposure to serum derived from patients with LC. Based on the results of bioinformatics and literature mining as shown in Fig. 2, the p38a kinase was identified as a potentially important factor in LC and was subject to further investigation. The in vivo experimental results confirmed that the use of whole genome methods to screen for target kinases is practical and effective.

In a previous study, the ultrastructure of BMECs in rats with LF was observed to be altered (22). Furthermore, vWF, an endothelial cell injury biomarker, was increased compared with normal controls (22). In the process of vascular endothelial damage, vWF mediates leukocyte and platelet adhesion (23). As the production of vWF is increased during vascular endothelial cell injury, it has been proposed to be an indicator of endothelial dysfunction (24). Consistent with these observations, the expression of vWF was observed to increase in the bone marrow smears from rats with LF compared with those of the controls in the current study. Additional studies involving a rat model of LF have also demonstrated an increased $\mathrm{vWF}$ expression in bone marrow smear tests $(8,9)$. Previous in vitro experiments have demonstrated that the serum from patients with LC may induce BMEC apoptosis (9). The in vivo results from the present study indicated that the apoptosis rate and the expression of caspase 3 in the bone marrow tissues of rats with LF were increased when compared with those of normal rats. These results suggest that LF may affect bone tissue survival and function, which may provide an explanation for the abnormal number of blood cells observed during LF. As an important component of the bone marrow microenvironment, BMECs support the growth and adhesion of hematopoietic cells, and secrete a number of cytokines to regulate hematopoietic cell functions. However, injury to, and apoptosis of, these cells may exacerbate the damage to bone marrow tissues during LC (25). Previous studies have demonstrated that p38a is highly expressed in BMECs during LC (9). p38a is an important member of the mitogen-activated protein kinase (MAPK) family, is involved in cell survival, differentiation and apoptosis, and is important in several cellular signaling pathways (26). Studies have demonstrated that p38a is closely associated with injury and apoptosis of the vascular endothelium. p38a regulates the production of tumor necrosis factor alpha (TNF $\alpha$ ), interleukin 1 (IL-1) and other inflammatory cytokines, and the subsequent signaling cascade leads to the release of a large amount of proinflammatory cytokines that may cause injury to endothelial cells (27). In addition, p38a is involved in the expression of the inducible isoform of nitric oxide synthase (iNOS) in endothelial cells, as well as the production of nitric oxide (28). Inhibiting this signaling pathway may reduce the production of iNOS and additional cytokines, as well as preventing the transport of the apoptotic protein BAX into mitochondria, thereby reducing injury to endothelial cells (28). In addition, p38a may promote apoptosis by activating the tumor suppressor gene p53, the proto-oncogene $c$-myc and additional important genes, including statl and PKB (29). Taking these observations into account, the authors hypothesize that the p38a kinase, which is highly expressed in BMECs during LC, may be an important factor in the injury and apoptosis of these cells. In support of this notion, Zhou et al (30) demonstrated that p38a was highly expressed in patients with bone marrow hypoplasia, and it was found to regulate apoptosis in hematopoietic stem cells. These results are consistent with those presented in the current study. 
Studies have indicated that specific cytokines may function as critical regulators of p38a expression in BMECs during LC. For example, transforming growth factor- $\beta$ (TGF $\beta$ ) has been observed to be an important factor that activates the p38-MAPK signaling pathway (31). Induction of TGF $\beta$ expression is one of the most important factors for promoting the onset of LC, and is maintained at a higher level in the blood during LC (32). Therefore, the blood environment during LC may provide favorable conditions for the activation of the p38-MAPK signaling pathway in BMECs. In addition, $\mathrm{TNF} \alpha$ is a potential factor that may activate p38a. Studies have demonstrated that serum TNF $\alpha$ levels in LC patients are significantly higher when compared with normal healthy individuals, and TNF $\alpha$ is reportedly capable of regulating bone repair following injury by activating p38a (33). Of particular note, TNF $\alpha$ and $\mathrm{p} 38 \mathrm{a}$ interact and activate caspase 3 to induce endothelial cell apoptosis (34). Nagila et al (35) demonstrated that $\mathrm{p} 38 \mathrm{a}$ inhibitors may reduce $\mathrm{TNF} \alpha$-induced apoptosis during dengue fever-induced liver damage. Therefore, TNF $\alpha$ may serve an important role in p38a-mediated BMEC apoptosis during LC. In addition, endotoxins may exacerbate bone marrow damage during LC (22). Portal hypertension during LC leads to reduced intestinal blood perfusion and damage to intestinal mucosa barrier function, which results in bacterial translocation $(25,36)$. As a consequence, a large quantity of intestinal toxins enter the blood, and lipopolysaccharide (LPS), the major component of endotoxin, may damage the vascular endothelial system and affect hematopoiesis in the bone marrow (37). However, whether TGF $\beta$, TNF $\alpha$, LPS or additional factors induce the high expression levels of $\mathrm{p} 38 \mathrm{a}$ kinase observed in BMECs during LC requires further investigation and verification.

In recent years, increased research has focused on protein kinases as therapeutic targets for a number of diseases, and the development of novel kinase inhibitors is growing rapidly. In addition, research into p38a inhibitors has progressed rapidly, and SB203580 is the most representative and widely used p38a inhibitor. Furthermore, compared with other $\mathrm{p} 38 \mathrm{a}$ inhibitors, SB203580 exhibits a more specific inhibitory effect (38). Therefore, SB203580 was employed as a p38a kinase inhibitor for the purposes of the present study. Following the administration of SB203580, vWF expression in bone marrow smear samples was significantly decreased compared with that of the LF group, which indicates that inhibition of $\mathrm{p} 38$ a kinase may reduce injury to the bone marrow endothelium during LF. In addition, the bone marrow tissue apoptotic rate and caspase 3 expression in the SB203580 group were significantly reduced compared with that of the control group, which suggests that p38a kinase may be an important factor that induces bone marrow tissue apoptosis during LF. Consistent with these results, Zhou et al (30) demonstrated that use of the p38a inhibitor may reduce hypoplastic bone marrow tissue apoptosis and stimulate hematopoiesis. The results of the present study associated with the application of SB203580 suggest the ability of p38a kinase to promote bone marrow tissue injury and apoptosis during LF, and the p38 kinase may therefore be a novel therapeutic target for treating the abnormal blood cell count observed during LC.

In conclusion, the present study performed whole genome and bioinformatics analyses to identify 14 differentially expressed genes that encode protein kinases in BMECs exposed to serum from patients with LC. Using a common bile duct ligation method, a rat model of LF was established successfully. Immunohistochemical analytical methods demonstrated that the expression levels of vWF, which is an indicator of endothelial cell injury, were significantly increased in the bone marrow smears of rats with LF compared with those of normal rats. In addition, the rate of apoptosis in rats with LF was also significantly increased compared with the controls. However, administration of the p38a inhibitor, SB203580, in rats with LF may improve these abnormal phenotypes to a certain degree. These results suggest that p38a may serve an important role in the damage and apoptosis of BMECs and bone marrow tissue during LF. However, further extensive experiments are required to elucidate the specific mechanisms involved in these processes.

\section{Acknowledgements}

This study was supported by the National Natural Science Foundation of China (grant nos. 81370566, 81170397, 81570579 and 81570581) and the Foundation of The First Affiliated Hospital of Harbin Medical University (grant no. 2011BS018).

\section{References}

1. Qamar AA, Grace ND, Groszmann RJ, Garcia-Tsao G, Bosch J, Burroughs AK, Ripoll C, Maurer R, Planas R, Escorsell A, et al: Incidence, prevalence, and clinical significance of abnormal hematological indices in compensated cirrhosis. Clin Gastroenterol Hepatol 7: 689-695, 2009.

2. Rois R, Sangro B, Herrero I, Quiroga J and Prieto J: The role of thrombopoietin in the thrombocytopenia of patients with live cirrhosis. Am J Gastroenterol 100: 1131-1136, 2005.

3. Sheehy T and Berman A: The anemia of cirrhosis. J Lab Clin Med 56: 72-82, 1960.

4. Mas VR, Fassnacht R, Archer KJ and Maluf D: Molecular mechanisms involved in the interaction effects of alcohol and hepatitis C virus in liver cirrhosis. Mol Med 16: 287-297, 2010.

5. Ramachandran R and Kakar S: Histological patterns in drug-induced liver disease. J Clin Pathol 62: 481-492, 2009.

6. Sullivan LW and Herbert V: Suppression of hematopoiesis by ethanol. J Clin Invest 43: 2048-2062, 1964.

7. Rosenfeld SJ and Young NS: Viruses and bone marrow failure. Blood Rev 5: 71-77, 1991.

8. Gao B, Wang D, Ma W, Jia X, Ma B, Zhang W and Xue D: MicroRNA-mRNA regulatory network study and apoptosis analysis on bone marrow endothelial cells induced by liver cirrhosis serum. Clin Res Hepatol Gastroenterol 38: 451-461, 2014.

9. Gao B, Sun W, Wang X, Jia X, Ma B, Chang Y, Zhang W and Xue D: Whole genome expression profiling and screening for differentially expressed cytokine genes in human bone marrow endothelial cells treated with humoral inhibitors in liver cirrhosis. Int J Mol Med 32: 1204-1214, 2013.

10. Kopp HG, Avecilla ST, Hooper AT and Rafii S: The bone marrow vascular niche: Home of HSC differentiation and mobilization. Physiology (Bethesda) 20: 349-356, 2005

11. Rafii S, Shapiro F, Rimarachin J, Nachman RL, Ferris B, Weksler B, Moore MA and Asch AS: Isolation and characterization of human bone marrow microvascular endothelial cells: Hematopoietic progenitor cell adhesion. Blood 84: 10-19, 1994.

12. Li WM, Huang WQ, Huang YH, Jiang DZ and Wang QR: Positive and negative haematopoietic cytokines produced by bone marrow endothelial cells. Cytokine 12: 1017-1023, 2000.

13. Hubbard SR and Till JH: Protein tyrosine kinase structure and function. Annu Rev Biochem 69: 373-398, 2000.

14. Manning G, Whyte DB, Martinez R, Hunter T and Sudarsanam S: The protein complement of the human genome. Science 298: 1912-1934, 2002.

15. Cheung CH, Coumar MS, Chang JY and Hsieh HP: Aurora kinase inhibitor patents and agents in clinical testing: An update (2009-10). Expert Opin Ther Pat 21: 857-884, 2011. 
16. Zempleni J and Mock DM: Chemical synthesis of biotinylated histones and analysis by sodium dodecyl sulfate-polyacrylamide gel electrophoresis/streptavidin-peroxidase. Arch Biochem Biophys 371: 83-88, 1999.

17. Chiu CT, Chen JH, Chou FP and Lin HH: Hibiscus sabdariffa leaf extract inhibits human prostate cancer cell invasion via down-regulation of Akt/NF-kB/MMP-9 pathway. Nutrients 7: 5065-5087, 2015.

18. Huang Y, Xia W, Lu M, Gao B, Qiao X, Sun B, Zhang W, Zhang Y and Xue D: Role of kinase epidermal growth factor receptor and SRC in the caerulein-induced acute pancreatitis in mice. Pancreas 44: 152-157, 2015.

19. Li Z, Ma B, Lu M, Qiao X, Sun B, Zhang W and Xue D Construction of network for protein kinases that play a role in acute pancreatitis. Pancreas 42: 607-613, 2013

20. Hofberger JA, Nsibo DL, Govers F, Bouwmeester K and Schranz ME: A complex interplay of tandem- and whole-genome duplication drives expansion of the L-type lectin receptor kinase gene family in the brassicaceae. Genome Biol Evol 7: 720-734, 2015

21. Draghetti C, Salvat C, Zanoguera F, Curchod ML, Vignaud C, Peixoto H, Di Cara A, Fischer D, Dhanabal M, Andreas G, et al: Functional whole-genome analysis identifies Polo-like kinase 2 and poliovirus receptor as essential for neuronal differentiation upstream of the negative regulator alphaB-crystallin. J Biol Chem 284: 32053-32065, 2009

22. Zhao S, Fu YM, Li XF, Jin ZF, Zhao RB, Huang Q, Zhang FM and Zhang WH: Alterations of bone marrow sinusoidal endothelium in rat and patients with liver cirrhosis. Dig Dis Sci 55 654-661, 2010

23. Wagner DD and Frenette PS: The vessel wall and its interactions Blood 111: 5271-5281, 2008.

24. Blann AD: von Willebrand factor and the endothelium in vascular disease. Br J Biomed Sci 50: 125-134, 1993.

25. Gao B, Li ZT, Xue DB and Zhang WH: A novel mechanism of abnormal hematological indices in liver cirrhosis: Bone marrow endothelial cell dysfunction caused by humoral inhibitor affects the hematopoietic function of bone marrow. Med Hypotheses 82: 282-285, 2014

26. Grossi V, Peserico A, Tezil T and Simone C: p38a MAPK pathway: A key factor in colorectal cancer therapy and chemoresistance. World J Gastroenterol 20: 9744-9758, 2014.

27. Ji RR, Gereau RW IV, Malcangio M and Strichartz GR: MAP kinase and pain. Brain Res Rev 60: 135-148, 2009.
28. Kevil CG, Oshima T and Alexander JS: The role of p38 MAP kinase in hydrogen peroxide mediated endothelial solute permeability. Endothelium 8: 107-116, 2001.

29. Kim SH, Kang JG, Kim CS, Ihm SH, Choi MG, Yoo HJ and Lee SJ: Apigenin induces c-Myc-mediated apoptosis in FRO anaplastic thyroid carcinoma cells. Mol Cell Endocrinol 369: 130-139, 2013.

30. Zhou L, Opalinska J and Verma A: p38 MAP kinase regulates stem cell apoptosis in human hematopoietic failure. Cell Cycle 6: 534-537, 2007.

31. Luo YH, Ouyang PB, Tian J, Guo XJ and Duan XC: Rosiglitazone inhibits TGF- $\beta 1$ induced activation of human Tenon fibroblasts via p38 signal pathway. PLoS One 9: e105796, 2014.

32. Gressner AM and Weiskirchen R: Modern pathogenetic concepts of liver fibrosis suggest stellate cells and TGF-beta as major players and therapeutic targets. J Cell Mol Med 10: 76-99, 2006.

33. Wang M, Crisostomo PR, Herring C, Meldrum KK and Meldrum DR: Human progenitor cells from bone marrow or adipose tissue produce VEGF, HGF, and IGF-I in response to TNF by a p38 MAPK-dependent mechanism. Am J Physiol Regul Integr Comp Physiol 291: R880-R884, 2006.

34. Yu J, Eto M, Akishita M, Okabe T and Ouchi Y: A selective estrogen receptor modulator inhibits TNF-alpha-induced apoptosis by activating ERK1/2 signaling pathway in vascular endothelial cells. Vascul Pharmacol 51: 21-28, 2009.

35. Nagila A, Netsawang J, Suttitheptumrong A, Morchang A, Khunchai S, Srisawat C, Puttikhunt C, Noisakran S, Yenchitsomanus PT and Limjindaporn T: Inhibition of p38MAPK and CD137 signaling reduce dengue virus-induced TNF- $\alpha$ secretion and apoptosis. Virol J 10: 105, 2013.

36. Douhara A, Moriya K, Yoshiji H, Noguchi R, Namisaki T, Kitade M, Kaji K, Aihara Y, Nishimura N, Takeda K, et al: Reduction of endotoxin attenuates liver fibrosis through suppression of hepatic stellate cell activation and remission of intestinal permeability in a rat non-alcoholic steatohepatitis model. Mol Med Rep 11: 1693-1700, 2015.

37. Meng F, Meliton A, Moldobaeva N, Mutlu G, Kawasaki Y, Akiyama T and Birukova AA: Asef mediates HGF protective effects against LPS-induced lung injury and endothelial barrier dysfunction. Am J Physiol Lung Cell Mol Physiol 308: L452-L463, 2015.

38. Bühler S and Laufer SA: p38 MAPK inhibitors: A patent review (2012-2013). Expert Opin Ther Pat 24: 535-554, 2014. 